\title{
Dependence of emission of terahertz radiation on geometrical parameters of dipole photoconductive antennas
}

\author{
Fumiaki Miyamaru, ${ }^{1,2, a)}$ Yu Saito, ${ }^{1,3}$ Kohji Yamamoto, ${ }^{4}$ Takashi Furuya, ${ }^{4}$ Seizi Nishizawa, ${ }^{1,5}$ \\ and Masahiko Tani ${ }^{4}$ \\ ${ }_{1}^{1}$ Department of Physics, Faculty of Science, Shinshu University, Asahi 3-1-1, Matsumoto, \\ Nagano 390-8621, Japan \\ ${ }^{2}$ PRESTO, Japan Science and Technology Agency, 2-1-1 Katahira, Aobaku-ku, Sendai 980-8577, Japan \\ ${ }^{3}$ Division of Environmental Science Development, Graduate School of Environmental Science, \\ Hokkaido University, Nishi 5, Kita hachi-jyo, Kita-ku, Sapporo 060-0808, Japan \\ ${ }^{4}$ Research Center for Development of Far-Infrared Region, University of Fukui, Bunkyo 3-9-1, \\ Fukui 910-8507, Japan \\ ${ }^{5}$ Advanced Infrared Spectroscopy Co., Ltd., 3-17-16, Sennin, Hachioji, Tokyo 193-0835, Japan
}

(Received 15 February 2010; accepted 5 May 2010; published online 24 May 2010)

\begin{abstract}
The dependence of the emission spectrum of terahertz $(\mathrm{THz})$ radiation on the geometrical parameters of the dipole antenna, and the relationship between these parameters and the temporal characteristics of the transient current that generate $\mathrm{THz}$ radiation, is reported. The emission intensity and the peak frequency strongly depend on these geometrical parameters. This dependence varies significantly with changes in the temporal characteristics of the transient current. We have also found that the emission efficiency can be increased by increasing the aspect ratio for the dipole. (C) 2010 American Institute of Physics. [doi:10.1063/1.3436724]
\end{abstract}

Terahertz $(\mathrm{THz})$ technologies have shown remarkable development during the past decade in various applications, e.g., medical diagnostics, ${ }^{1-3}$ security applications, ${ }^{4-6}$ and information technology. ${ }^{7-9}$ The photoconductive antennas typically used for emitting $\mathrm{THz}$ radiation consist of metallic antenna patterns fabricated onto semiconductor substrates. ${ }^{10}$ For the practical $\mathrm{THz}$ applications mentioned above, the high emission intensity of $\mathrm{THz}$ radiation is crucial for increasing the signal-to-noise $(\mathrm{S} / \mathrm{N})$ ratio of the spectroscopic system. The optimization of the photoconductive antenna's design ${ }^{11-15}$ is intended to increase the intensity of the $\mathrm{THz}$ radiation.

In the early period of $\mathrm{THz}$ research, the relationship between the emission spectra from the photoconductive antenna and the antenna's design was investigated. Smith et al. ${ }^{16}$ reported the dependence of the $\mathrm{THz}$ emission spectra on dipole antennas having three different lengths. They found that the emission intensity significantly decreases as the dipole length decreases from 200 to $50 \mu \mathrm{m}$. The temporal dynamics of the transient current, induced by illuminating the photoconductive antenna with a femtosecond laser pulse, is also a crucial parameter for the emission characteristics of the $\mathrm{THz}$ radiation. The dependence of the emission spectrum on the pulse duration of the femtosecond laser was reported by Duvillaret et al. ${ }^{17}$ Nevertheless, of the many efforts to investigate the emission characteristics of photoconductive antennas, none have focused on the relationship between the geometric parameters of the antenna design and the temporal characteristics of the transient current. In order to optimize the antenna design for individual, practical applications, this relationship is a very important issue.

Here, we report the results of our investigation of the dependence of the emission spectrum of $\mathrm{THz}$ radiation on the geometrical parameters of the dipole antenna, and the

\footnotetext{
${ }^{\text {a) }}$ Author to whom correspondence should be addressed. Electronic mail: miyamaru@shinshu-u.ac.jp.
}

relationship between these parameters and the temporal characteristics of the transient current that generates $\mathrm{THz}$ radiation. In our experiments, the intensity of the $\mathrm{THz}$ radiation significantly increased with increasing dipole length. Additionally, the emission efficiency of the $\mathrm{THz}$ radiation strongly depends on the relative dimensions of the dipole; efficiency increases significantly with increasing aspect ratios $\eta(\eta \equiv L / W)$. These results are valuable for optimizing the photoconductive antenna design.

Photoconductive antennas with dipole patterns were fabricated on low-temperature-grown gallium arsenide (LT-GaAs) substrates using a lithographic technique. The bottom inset of Fig. 1 shows a schematic picture of a photoconductive antenna used in our experiment. We investigated the dependence of the $\mathrm{THz}$ radiation characteristics on two geometrical parameters of the dipole pattern: the length $L$

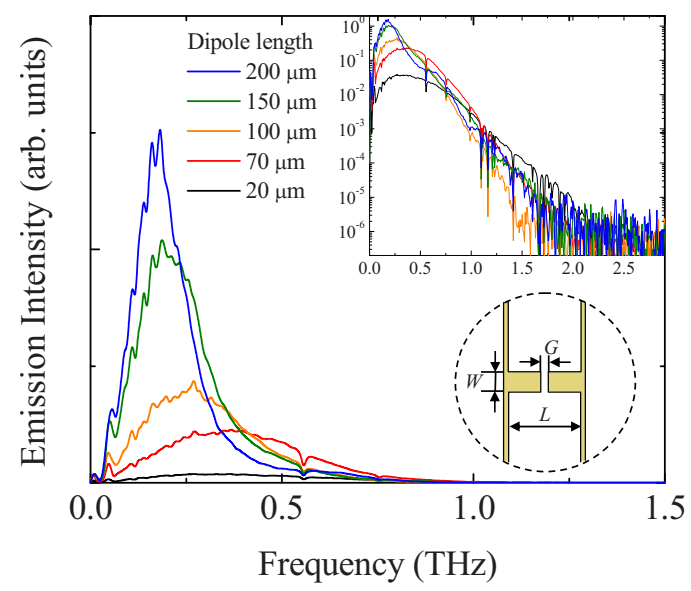

FIG. 1. (Color online) Measured emission spectra from photoconductive dipole antennas with dipole lengths ranging from 20 to $200 \mu \mathrm{m}$. Upper inset shows the same spectra with logarithmic scale of the vertical axis. Lower inset shows the schematic of the photoconductive dipole antenna used. 
and width $W$ of the dipole line. The $5 \mu \mathrm{m}$ wide gap fabricated at the center of the dipole line allows for the excitation of the transient current using a femtosecond laser pulse. The $\mathrm{THz}$ radiation emission spectra for each of the photoconductive antennas were measured by $\mathrm{THz}$ time-domain spectroscopy (THz-TDS). ${ }^{18} \mathrm{THz}$ radiation was generated using $\tau_{0}=120$ fs laser pump pulses at $800 \mathrm{~nm}$. The THz radiation was detected by a dipole-type photoconductive antenna, gated with time-delayed probe laser pulses. The length $L$ and the width of the dipole antenna for the detector were fixed at $L=70 \mu \mathrm{m}$ and $W=10 \mu \mathrm{m}$, respectively. By changing the delay-time between the pump and probe laser pulses, the time dependent amplitude of the $\mathrm{THz}$ radiation was recorded. The measured wave form of the $\mathrm{THz}$ radiation was converted into a power intensity spectrum by Fourier transform.

First we investigated the dependence of the emission spectra on the dipole length. Figure 1 shows the measured emission spectra of the $\mathrm{THz}$ radiation from dipole antennas with several dipole lengths. In these experiments, the dipole length was varied from 20 to $200 \mu \mathrm{m}$. For $L=20 \mu \mathrm{m}$ (black line), a very broad emission spectra is observed, with a peak frequency of $0.35 \mathrm{THz}$. The peak intensity of the emission spectra significantly increases as the dipole length increases. For $L=200 \mu \mathrm{m}$ (blue line), the peak intensity is about 35 times larger than that for $L=20 \mu \mathrm{m}$. This result agrees well with the results reported by Smith et al. ${ }^{16}$ With increasing dipole length, the peak frequency of the emission spectra shifts to lower frequency and reaches $0.25 \mathrm{THz}$ for $L$ $=200 \mu \mathrm{m}$. At frequencies higher than $1.0 \mathrm{THz}$, the emission spectrum for $L=20 \mu \mathrm{m}$ shows the highest intensity when compared with other antennas (see the inset of Fig. 1). This means that the frequency bandwidth becomes narrower as the dipole length increases.

In order to explain the experimental results shown in Fig. 1, we calculated the theoretical emission spectra. In these calculations, we utilized the finite-difference timedomain method. To generate $\mathrm{THz}$ radiation, the transient electric field is applied at the gap of the dipole antenna. The time variation in this input electric field is mainly determined by two experimental parameters: the pulse width of an illuminated femtosecond laser pulse $\tau_{0}$ and the decay time of the photoexcited carriers $\tau_{\mathrm{c}}$ in LT-GaAs substrates. ${ }^{19}$ We calculated the emitted electric field $E(t)$ which is proportional to the time derivation of the current $\dot{J}(t)$ at the gap by using these two parameters. Figures 2(a)-2(c) show the emission spectra for several dipole antennas of varying lengths, for $\tau_{0}$ of $200 \mathrm{fs}, 120 \mathrm{fs}$, and $80 \mathrm{fs}$, respectively. The decay time of the photoexcited carriers is taken to be $\tau_{\mathrm{c}}=500 \mathrm{fs}$ for all calculations. The spectrum of electric field without dipole antenna is shown as a reference (dashed black curve), and intensities were multiplied by 100 for clarity. In Fig. 2(a), the peak intensity of the emission spectrum increases monotonically and the peak frequency shifts to lower frequency as the dipole length increases. This result is in good agreement with that observed in the experiment. On the other hand, in Figs. 2(b) and 2(c), the emission spectra show dependences on the dipole length that are different from that shown in Fig. 2(a). In Fig. 2(b), although the peak intensity is still the largest for the dipole length $L=200 \mu \mathrm{m}$, the peak intensities of shorter dipole lengths $(L=20$ and $70 \mu \mathrm{m})$ become much larger as the result of the shorter laser pulse width. The peak frequency shifts to lower frequency when varying the dipole

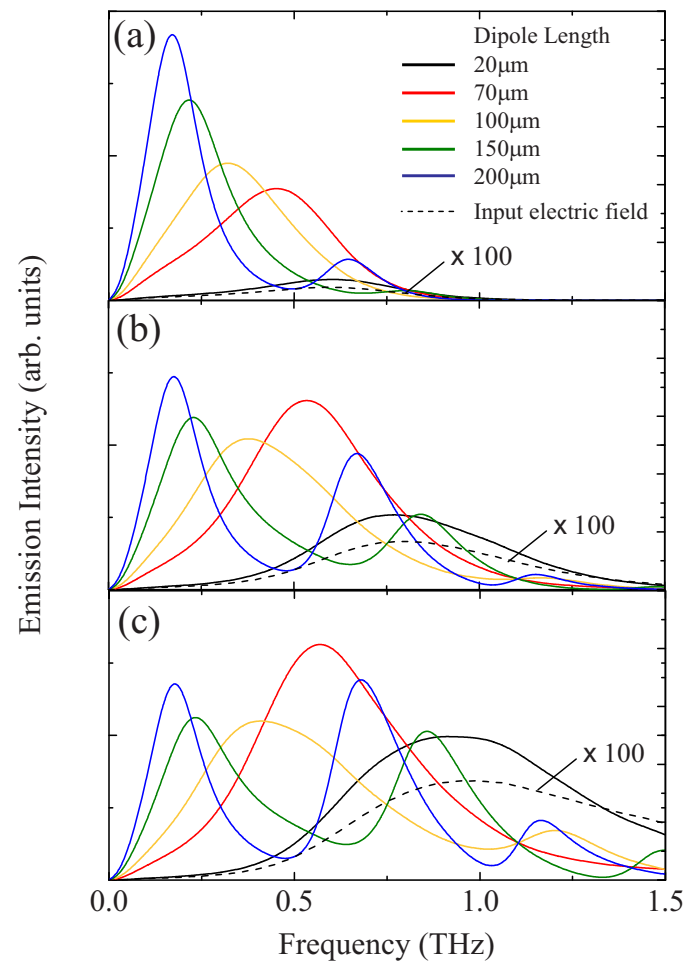

FIG. 2. (Color online) Calculated emission spectra from dipole antennas with dipole lengths ranging from 20 to $200 \mu \mathrm{m}$. For (a)-(c), we used the different input electric fields whose emission spectra are shown in each figure (dashed line).

length from $L=20$ to $200 \mu \mathrm{m}$. This trend is similar to that observed in Fig. 2(a). However, in Fig. 2(b) the second order resonant peaks are clearly observed at $0.71 \mathrm{THz}$ and 0.84 $\mathrm{THz}$ for dipole lengths $L=200 \mu \mathrm{m}$ and $150 \mu \mathrm{m}$, respectively. These second order peaks appear more clearly in Fig. 2(c), for which the shortest laser pulse width was assumed.

The simulated results shown in Figs. 2(a)-2(c) can be attributed to the fact that the relationship between the geometrical parameters of the dipole antenna and the spectral shape of the input electric field determines the characteristics of the $\mathrm{THz}$ emission spectrum. When the input electric field changes relatively slowly, and correspondingly has a lower frequency spectrum [Fig. 2(a)], the maximum emission intensity can be obtained using a longer dipole length which has a resonance at a lower frequency. Our experiment is corresponding to this case. When the input electric field has a higher frequency spectrum, the maximum peak intensity can be obtained with a shorter dipole as is shown in Fig. 2(c). In this case, the resonant frequency of the dipole antenna is close to the peak frequency of the input electric field. Even for longer dipole lengths, large peak intensities can be obtained if the second resonant frequency is close to the peak frequency of the input electric field. We note that the simulation results shown in Fig. 2(a) agree with the experimental results shown in Fig. 1 although the laser pulse widths are different in the two cases $\left[\tau_{0}=200\right.$ fs for Fig. 2(a) and 120 fs for Fig. 1]. This might be because we did not include the effect of the detector's antenna in our calculation.

In order to obtain large emission intensities, the dipole width $W$ (see the inset of Fig. 1) is also an important parameter. Figure 3(a) shows the calculated emission spectra from dipole antennas having dipole widths varying from $W=10$ to $200 \mu \mathrm{m}$. The dipole length is fixed at $L=30 \mu \mathrm{m}$, and the 

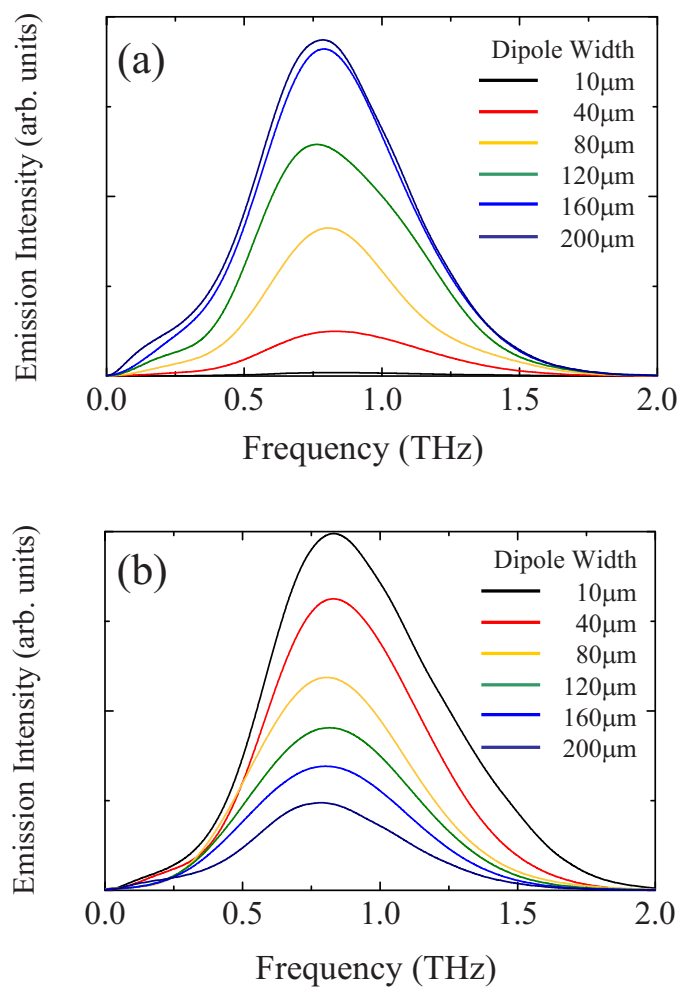

FIG. 3. (Color online) Calculated emission spectra from dipole antennas having dipole widths varying from 10 to $200 \mu \mathrm{m}$. (a) Intensity of the input electric field increases proportionally with the dipole width. (b) Intensity of the input electric field is constant for all dipole widths.

input electric field for all calculations is the same as that used for the calculations shown in Fig. 2(b). The peak intensity increases monotonically with increasing the dipole width and has a maximum value for $W=200 \mu \mathrm{m}$. This implies that the dipole width should be larger in order to obtain high emission intensity when sufficient power is provided by the laser to pump the overall photoconductive gap with a width $W$. Figure 3(b) shows the calculated emission spectra obtained from the same dipole antennas used in Fig. 3(a) but with a constant total input current. Contrary to the previous case [Fig. 3(a)], the peak intensity decreases significantly with increasing dipole width. This indicates that the emission efficiency of the dipole antenna decreases as the aspect ratio $\eta(\equiv L / W)$ of the dipole antenna decreases.

In summary, we have investigated the dependence of the emission spectrum of the $\mathrm{THz}$ radiation on the geometrical parameters of the photoconductive dipole antenna. We have found that the emission intensity and the peak frequency strongly depend on the length and width of the dipoles. Such dependences are closely related to the spectral characteristics of the input electric field and correspondingly the transient current. We have also found that the emission efficiency can be increased by increasing the aspect ratio of the dipole. We believe that these results are very important for the optimization of the design of the photoconductive dipole antenna.

This work was supported in part by Development of Systems and Technology for Advanced Measurement and Analysis, Japan Science and Technology Agency. F.M. is also supported by the Ministry of Education, Science, Sports and Culture, Grant-in-Aid for Young Scientists (B) under Grant No. 21760036.

${ }^{1}$ K. J. Siebert, H. Quast, R. Leonhardt, T. Löffler, M. Thomson, T. Bauwe, H. G. Roskos, and S. Czasch, Appl. Phys. Lett. 80, 3003 (2002).

${ }^{2}$ S. Nakajima, H. Hoshina, M. Yamashita, C. Otani, and N. Miyoshi, Appl. Phys. Lett. 90, 041102 (2007).

${ }^{3}$ H. Hoshina, A. Hayashi, N. Miyoshi, F. Miyamaru, and C. Otani, Appl. Phys. Lett. 94, 123901 (2009).

${ }^{4}$ K. Yamamoto, M. Yamaguchi, F. Miyamaru, M. Tani, and M. Hangyo, Jpn. J. Appl. Phys., Part 2 43, L414 (2004).

${ }^{5}$ N. Karpowicz, H. Zhong, C. Zhang, K.-I. Lin, J.-S. Hwang, J. Xu, and X.-C. Zhang, Appl. Phys. Lett. 86, 054105 (2005).

${ }^{6}$ H.-B. Liu, Y. Chen, G. J. Bastiaans, and X.-C. Zhang, Opt. Express 14, 415 (2006).

${ }^{7}$ C. M. Mann, in Terahertz Sources and Systems, edited by R. E. Miles, P. Harrison, and D. Lippens (Kluwer, Dordrecht, 2001), p. 261.

${ }^{8}$ P. H. Siegel, IEEE Trans. Microwave Theory Tech. 50, 910 (2002).

${ }^{9}$ M. Tonouchi, Nat. Photonics 1, 97 (2007).

${ }^{10}$ D. H. Auston, K. P. Cheung, and P. R. Smith, Appl. Phys. Lett. 45, 284 (1984).

${ }^{11}$ M. van Exter, C. Fattinger, and D. Grischkowsky, Appl. Phys. Lett. 55, 337 (1989).

${ }^{12}$ M. Tani, S. Matsuura, K. Sakai, and S. Nakashima, Appl. Opt. 36, 7853 (1997).

${ }^{13}$ R. Mendis, C. Sydlo, J. Sigmund, M. Feiginov, P. Meissner, and H. L. Hartnagel, Solid-State Electron. 48, 2041 (2004).

${ }^{14}$ M. Awad, M. Nagel, H. Kurz, J. Herfort, and K. Ploog, Appl. Phys. Lett. 91, 181124 (2007).

${ }^{15}$ Y. Hirota, R. Hattori, M. Tani, and M. Hangyo, Opt. Express 14, 4486 (2006).

${ }^{16}$ P. R. Smith, D. H. Auston, and M. C. Nuss, IEEE J. Quantum Electron. 24, 255 (1988).

${ }^{17}$ L. Duvillaret, F. Garet, J.-F. Roux, and J.-L. Coutaz, IEEE J. Sel. Top. Quantum Electron. 7, 615 (2001).

${ }^{18}$ M. Hangyo, T. Nagashima, and S. Nashima, Meas. Sci. Technol. 13, 1727 (2002).

${ }^{19}$ S. Hughes, M. Tani, and K. Sakai, J. Appl. Phys. 93, 4880 (2003). 